\title{
Interference at the Single Photon Level Along Satellite-Ground Channels
}

\author{
Giuseppe Vallone, ${ }^{1}$ Daniele Dequal, ${ }^{1}$ Marco Tomasin, ${ }^{1}$ Francesco Vedovato, ${ }^{1}$ Matteo Schiavon, ${ }^{1}$ \\ Vincenza Luceri, ${ }^{2}$ Giuseppe Bianco, ${ }^{3}$ and Paolo Villoresi ${ }^{1, *}$ \\ ${ }^{1}$ Dipartimento di Ingegneria dell'Informazione, Università degli Studi di Padova, Padova 35131, Italy \\ ${ }^{2} e$-GEOS spa, Matera 75100 , Italy \\ ${ }^{3}$ Matera Laser Ranging Observatory, Agenzia Spaziale Italiana, Matera 75100, Italy \\ (Received 18 January 2016; published 21 June 2016)
}

\begin{abstract}
Quantum interference arising from the superposition of states is striking evidence of the validity of quantum mechanics, confirmed in many experiments and also exploited in applications. However, as for any scientific theory, quantum mechanics is valid within the limits in which it has been experimentally verified. In order to extend such limits, it is necessary to observe quantum interference in unexplored conditions such as moving terminals at large distances in space. Here, we experimentally demonstrate single photon interference at a ground station due to the coherent superposition of two temporal modes reflected by a rapidly moving satellite a thousand kilometers away. The relative speed of the satellite induces a varying modulation in the interference pattern. The measurement of the satellite distance in real time by laser ranging allows us to precisely predict the instantaneous value of the interference phase. We then observed the interference patterns with a visibility up to $67 \%$ with three different satellites and with a path length up to $5000 \mathrm{~km}$. Our results attest to the viability of photon temporal modes for fundamental tests of physics and quantum communication in space.
\end{abstract}

DOI: 10.1103/PhysRevLett.116.253601

Introduction.-Quantum interference has played a crucial role to highlight the essence of quantum mechanics since the Einstein-Bohr dialogues at the end of the 1920s [1]. Indeed, it originates when alternative possibilities in a quantum process are indistinguishable, like in the case of individual particles that may be simultaneously in more than one place as in the well-known Young double-slit experiment [1]. Quantum interference has been observed with photons [2,3], but also with electrons [4], with neutrons [5], and even with large molecules with masses exceeding $10000 \mathrm{amu}$ [6]. One of the main challenges in quantum physics is establishing if fundamental bounds to interference exist: for instance, can quantum interference be measured by observers in relative motion and at an arbitrary large distance? To our knowledge, the longest path on which interference at the single photon level was tested is a $307 \mathrm{~km}$ fiber link on the ground with a fixed sender and receiver [7]. Classical interference in free space has been observed in gravitational wave detectors based on a Michelson interferometer with $4 \mathrm{~km}$ long arms and using a laser beam with kilowatt power [8].

Here, we demonstrate interference at the single photon level along satellite-ground channels by exploiting temporal modes of single photons. To this purpose, we exploited a coherent superposition between two single-photon wave packets on the ground and observed their interference after the reflection by a rapidly moving satellite at a very large distance with a total path length up to $5000 \mathrm{~km}$. The varying relative velocity of the satellite with respect to the ground introduces a modulation in the interference pattern that can be predicted by special relativistic calculations, as explained below.

Description of the experiment.-In our scheme, a coherent state $\left|\Psi_{\text {out }}\right\rangle$ in two temporal modes is generated at the

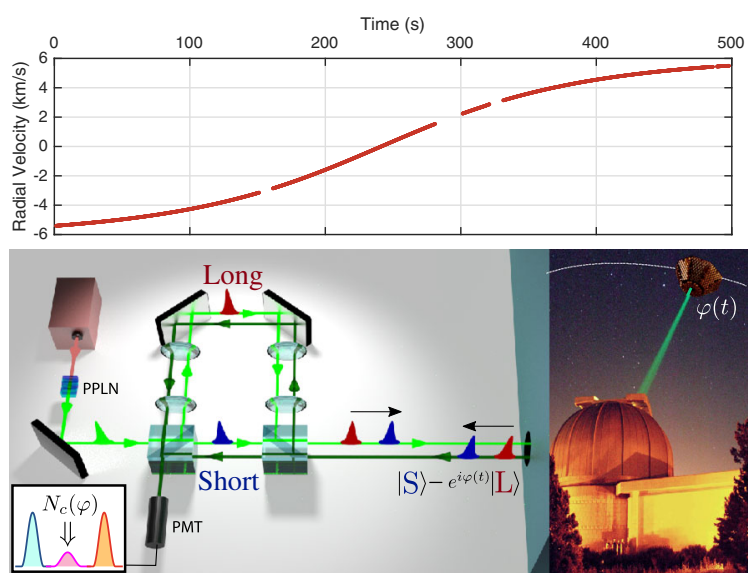

FIG. 1. Scheme of the experiment and satellite radial velocity. In the top panel we show the measured radial velocity of the Beacon-C satellite ranging from -6 to $+6 \mathrm{~km} / \mathrm{s}$ as a function of time during a single passage. In the bottom panel we show the unbalanced MZI with the two $4 f$ systems used for the generation of the state and the measurement of the interference. The light and dark green lines, respectively, represent the beams outgoing to and ingoing from the telescope. In the inset, we show the expected detection pattern: the number of counts $N_{c}$ in the central peak varies according to the kinematic phase $\varphi$ imposed by the satellite. The right photo shows the MLRO with the laser ranging beam and the Beacon-C satellite (not to scale). The phase $\varphi(t)$ depends on the satellite radial velocity as described in the text. 
ground station with an unbalanced Mach-Zehnder interferometer (MZI), sketched in the lower panel of Fig. 1. The delay $\Delta t \simeq 3.4 \mathrm{~ns}$ between the wave packets of the two modes corresponds to a length difference between the two arms of $\ell=c \Delta t \simeq 1 \mathrm{~m}$ ( $c$ is the speed of light in vacuum) and it is much longer than the coherence time $\tau_{c} \approx 83$ ps of each wave packet (we used the convention of Ref. [9] for the definition of $\tau_{c}$ ). Using a telescope, the state $\left|\Psi_{\text {out }}\right\rangle$ is directed to a retroreflector placed on a satellite in orbit. The satellite retroreflectors redirect the beam back to the ground station, where it is collected and injected into the same MZI used in the uplink. After the reflection by the satellite and the downlink attenuation, the state collected by the telescope can be written as $\left|\Psi_{r}\right\rangle=(1 / \sqrt{2})\left(|S\rangle-e^{i \varphi(t)}|L\rangle\right)$, namely, as a a superposition of two single-photon wave packets $|S\rangle$ and $|L\rangle$ (the quantum state written above corresponds to the renormalized single-photon part of the state received at the telescope). We note that the above superposition is also known as time-bin encoding, and it is used for fundamental tests of quantum mechanics [10-13], for quantum information applications such as quantum key distribution [14,15] along optical fibers [16-18], and for increasing the dimension of the Hilbert space in which information can be encoded [19].

As we now explain, the relative phase $\varphi(t)$ is determined by the satellite instantaneous radial velocity with respect to the ground, $v_{r}(t)$. Indeed, at a given instant $t$, the satellite motion determines a shift $\delta r(t)$ of the reflector radial position, during the separation $\Delta t$ between the two wave packets. This shift can be estimated at first order as $\delta r(t) \approx v_{r}(t) \Delta t$, and its value may reach a few tens of micrometers for the satellites here used. For instance, in the top panel of Fig. 1, we show the value of $v_{r}(t)$, which ranges from -6 to $6 \mathrm{~km} / \mathrm{s}$ for the selected passage of the Beacon-C satellite. Therefore, the satellite motion imposes during reflection the additional kinematic phase $\varphi(t) \approx$ $2 \delta r(t)(2 \pi / \lambda)$ between the wave packets $|L\rangle$ and $|S\rangle$, where $\lambda$ is the pulse wavelength in vacuum (see Fig. 2).

A single MZI for state generation and detection intrinsically ensures the same unbalance of the arms and avoids active stabilization, necessary otherwise with two independent interferometers. As detailed in the Supplemental Material [20], two $4 f$ systems realizing an optical relay equal to the arm length difference were placed in the long arm of the MZI. The relay is required to match the interfering beam wave fronts that are distorted by the passage through atmospheric turbulence: otherwise, the latter may cause distinguishability between the two paths, washing out the interference. The MZI at the receiver is able to reveal the interference between the two returning wave packets. At the MZI outputs we expect detection times that follow the well known three-peak profile (see Fig. 1): the first peak represents the pulse $|S\rangle$ taking again the short arm, while the third represents the delayed pulse $|L\rangle$ taking again the long arm. In the central peak we expect

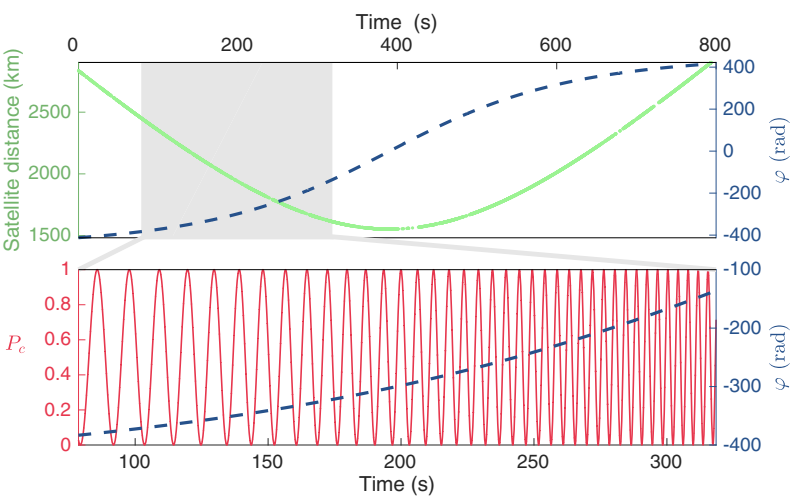

FIG. 2. Kinematic phase and interference pattern. Top panel: we show the measured satellite distance and the predicted kinematic phase $\varphi(t)$ estimated by Eq. (2) as a function of time for a passage of the Ajisai satellite. The shaded area represents the temporal window of data acquisition. Bottom panel: kinematic phase $\varphi(t)$ and theoretical probability $P_{c}(t)$ in the shaded area. The interference pattern is modulated according to the value of $\varphi(t)$ determined by the satellite velocity.

indistinguishably between two alternative possibilities: the $|S\rangle$ pulse taking the long arm and the $|L\rangle$ pulse taking the short arm in the path along the MZI toward the detector. The signature of the interference at the single photon level is then obtained when the counts in the central peak differ from the sum of the counts registered in the lateral peaks.

To measure the interference we used a single photon detector [photomultiplier tube (PMT)] placed at the available port of the MZI, as shown in Fig. 1. For a moving retroreflector, as detailed in the Supplemental Material [20], special relativistic calculations show that the probability $P_{c}$ of detecting the photon in the central peak is given by

$$
P_{c}(t)=\frac{1}{2}[1-\mathcal{V}(t) \cos \varphi(t)]
$$

with

$$
\varphi(t)=\frac{2 \beta(t)}{1+\beta(t)} \frac{2 \pi c}{\lambda} \Delta t, \quad \mathcal{V}(t)=e^{-\frac{\lambda^{2} \varphi^{2}(t)}{8 \pi c^{2} \tau_{c}^{2}}} \simeq 1
$$

We note that for a retroreflector at rest we expect $P_{c}=0$. The parameter $\beta(t)$ is defined as $\beta(t)=v_{r}(t) / c$. The above relation is obtained by time-of-flight calculations together with the Doppler effect that changes the angular frequency of the reflected pulses from $\omega_{0} \equiv 2 \pi c / \lambda$ to $\omega_{0}(1-\beta) /(1+\beta)$. We note that the first order approximation of Eq. (2) gives the phase $\varphi(t) \approx 4 \pi v_{r}(t) \Delta t / \lambda$ above described. The theoretical visibility $\mathcal{V}(t)$ is approximately 1 since the $\beta$ factor is upper bounded by $3 \times 10^{-5}$ in all the experimental studied cases, while the ratio $\Delta t / \tau_{c}$ is of the order of $10^{2}$.

Experimental results.-We realized our experiment at the Matera Laser Ranging Observatory (MLRO) of the Italian 
Space Agency in Matera, Italy, which is equipped with a $1.5 \mathrm{~m}$ telescope designed for precise satellite tracking and which acted as a ground quantum hub for the first demonstrations of space quantum communication $[21,22]$. The pulses used to prepare $\left|\Psi_{\text {out }}\right\rangle$ are generated by a modelocking laser based on a $\mathrm{Nd}: \mathrm{YVO}_{4}$ gain medium operating at a repetition rate stabilized at $100 \mathrm{MHz}$ by an atomic clock and at the wavelength of $1064 \mathrm{~nm}$. Each pulse is upconverted with a Periodically Poled Lithium Niobate (PPLN) crystal to a wavelength of $532 \mathrm{~nm}$ and an energy of $\sim 1 \mathrm{~nJ}$. The pulses, after the MZI, are sent to the Coudé path of the MLRO telescope, which directs the state $\left|\Psi_{\text {out }}\right\rangle$ toward the satellite while actively tracking its orbit. We selected three satellites in low Earth orbit-Beacon-C, Stella, and Ajisai-which are equipped with efficient corner-cube retroreflectors. Thanks to the corner-cube retroreflector properties, the state is automatically redirected toward the ground station, where it is injected into the same MZI used in the uplink.

The value of $\varphi(t)$ originating from the satellite motion can be precisely predicted on the basis of the sequence of measurements of the instantaneous distance of the satellite, or range $r$, which is realized in parallel. The range is measured by a strong satellite laser ranging (SLR) signal at $10 \mathrm{~Hz}$ and energy per pulse of $100 \mathrm{~mJ}$. Thanks to an atomic clock, the SLR pulses are separated precisely by $\Delta T=$ $100 \mathrm{~ms}$ and synchronized with the $100 \mathrm{MHz}$ pulses. By measuring the temporal separation $\Delta T^{\prime}$ of the SLR pulses at the receiver after the satellite retroreflection, it is possible to determine the instantaneous satellite velocity relative to the ground station $v_{r}(t)$. Indeed, since by the Doppler effect $\Delta T^{\prime}=\Delta T(1+\beta) /(1-\beta)$, the velocity $v_{r}(t)$ can be estimated as $v_{r}(t)=c\left(\Delta T^{\prime}-\Delta T\right) /\left(\Delta T^{\prime}+\Delta T\right)$. The separation $\Delta T^{\prime}$ is related to the range $r$ by $\Delta T^{\prime}=\Delta T+\Delta r / c$, where $\Delta r$ is the variation of the satellite distance between two subsequent SLR pulses. Then, by measuring the range every $100 \mathrm{~ms}$, the instantaneous satellite velocity relative to the ground station $v_{r}(t)$ can be estimated, from which $\varphi(t)$ can be derived by Eq. (2). In the top panel of Fig. 2, for a given passage of the Ajisai satellite, we show the measured satellite distance and the estimated $\varphi(t)$ as a function of time from the beginning to the end of the satellite tracking. Since $v_{r}(t)$ is continuously changing along the orbit, the value of $\varphi(t)$ is varying accordingly. In the bottom panel of Fig. 2 we show the variation of the theoretical output probability $P_{c}(t)$ along the Ajisai orbit as predicted by Eq. (1).

By the synchronization technique described in the Supplemental Material [20], we determined the expected $\left(t_{\text {ref }}\right)$ and the measured $\left(t_{\text {meas }}\right)$ instant of arrival of each photon. In this way, the histogram of the detections in the temporal window of $10 \mathrm{~ns}$ between two consecutive pulses as a function of the temporal difference $\Delta=t_{\text {meas }}-t_{\text {ref }}$ can be obtained. In Fig. 3 we show such histograms corresponding to constructive and destructive interference in the case of satellite Beacon-C.

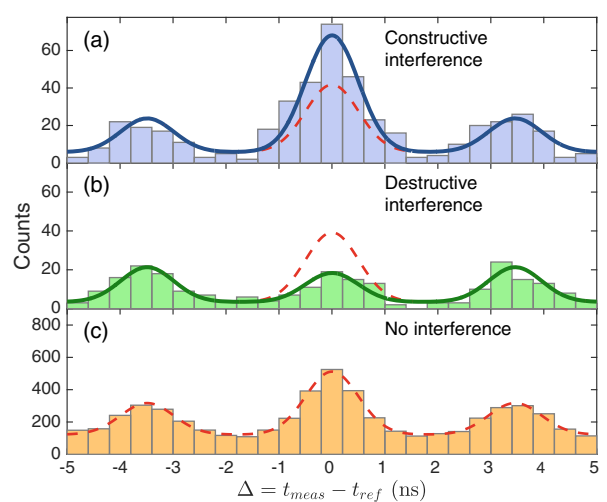

FIG. 3. Constructive and destructive single photon interference [Beacon-C satellite, 11.07.2015 h 1.33 central european summer time (CEST)]. (a) Histogram of single photon detections as a function of time $\Delta=t_{\text {meas }}-t_{\text {ref }}$ realized by selecting only the returns characterized by $\varphi(\bmod 2 \pi) \in[4 \pi / 5,6 \pi / 5]$ that lead to constructive interference. The solid line shows the tri-Gaussian fit. By evaluating the Gaussian integrals we obtained the counts $N_{\ell}=112 \pm 11$ for the sum of lateral peaks and $N_{c}=196 \pm 14$ for the central one. (b) Histogram of single photon detections realized by selecting only the returns characterized by $\varphi(\bmod 2 \pi) \in[-\pi / 5, \pi / 5]$. Here, $N_{\ell}=112 \pm 11$ and $N_{c}=$ $46 \pm 7$. (c) Histogram of single photon detections without any selection on the phase. As expected, the interference is completed washed out and we measured $N_{c}=1245 \pm 35$ and $N_{\ell}=$ $1306 \pm 36$, fully compatible with $P_{c}=1 / 2$. In all panels, the dotted red lines represent the expected counts in case of no interference.

In particular, for the constructive interference, Fig. 3(a), we selected the detections corresponding to $\varphi(\bmod 2 \pi) \in$ $[4 \pi / 5,6 \pi / 5]$. For the destructive interference, Fig. 3(b), we selected a kinematic phase $\varphi(\bmod 2 \pi) \in[-\pi / 5, \pi / 5]$. The detections in the central peak are, respectively, higher or lower than the sum of the two lateral peaks in the two cases. We note that the peak width is determined by the detector timing jitter, which has a standard deviation $\sigma=0.5 \mathrm{~ns}$. These two histograms clearly show the interference effect in the central peak. On the contrary, Fig. 3(c) is obtained by taking all the data without any selection on $\varphi$. In this case, the interference is completely washed out. These results show that, in order to prove the interference effect, it is crucial to correctly predict the kinematic phase $\varphi$ imposed by the satellite motion.

By using the data of Fig. 3, we experimentally evaluate the probability $P_{c}^{(\exp )}$ as the ratio of the detections associated with the central peak $N_{c}$ to twice the sum $N_{\ell}$ of the detections associated with the side peaks, namely,

$$
P_{c}^{(\exp )}=\frac{N_{c}}{2 N_{\ell}} .
$$

The values $P_{c}^{(\exp )}=0.87 \pm 0.10$ and $P_{c}^{(\exp )}=0.20 \pm 0.03$ are obtained for constructive and destructive interference, respectively. The values deviate with clear statistical 


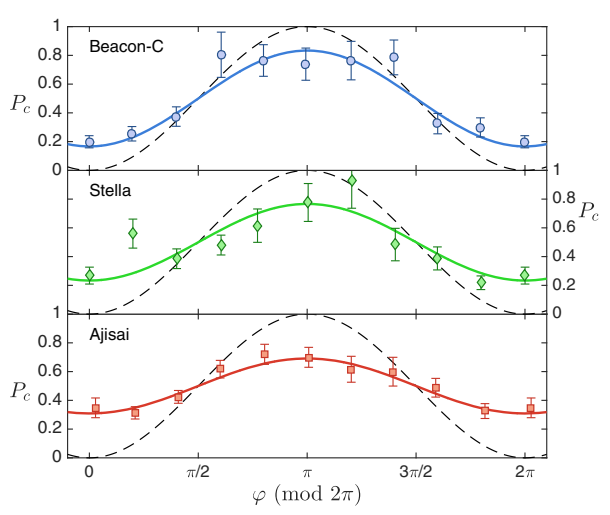

FIG. 4. Experimental interference pattern. Experimental probabilities $P_{c}^{(\exp )}$ as a function of the kinematic phase measured for three different satellites. By fitting the data we estimate the visibilities $\mathcal{V}_{\exp }=67 \pm 11 \%$ for Beacon-C, $\mathcal{V}_{\exp }=53 \pm 13 \%$ for Stella, and $\mathcal{V}_{\exp }=38 \pm 4 \%$ for Ajisai. The dashed lines correspond to the theoretical value of $P_{c}$ predicted by Eq. (1). The points are obtained by considering ten intervals of the phase defined by $\mathcal{I}_{j} \equiv[(j-1 / 10) \pi,(j+1 / 10) \pi]$. For each interval we selected the data corresponding to $\varphi(\bmod 2 \pi) \in \mathcal{I}_{j}$ : from such data we determined the experimental probability of detection in the central peak $P_{c}^{(\exp )}$ and we averaged the corresponding phase $\varphi$. We note that at the point $\varphi=0$ and $\varphi=2 \pi$ the same subset of data was selected.

evidence from 0.5 , which is the expected value in the case of no interference.

More clear evidence of the role of $\varphi(t)$ can be demonstrated by evaluating the experimental probabilities $P_{c}^{(\exp )}$ as a function of $\varphi$. Figure 4 shows $P_{c}^{(\exp )}$ for ten different values of the kinematic phase $\varphi$ and for the three different satellites. By fitting the data by $P_{c}^{(\exp )}=\frac{1}{2}\left(1-\mathcal{V}_{\exp } \cos \varphi\right)$, we estimated the experimental visibilities $\mathcal{V}_{\exp }=67 \pm 11 \%$ for Beacon-C, $\mathcal{V}_{\exp }=53 \pm 13 \%$ for Stella, and $\mathcal{V}_{\exp }=$ $38 \pm 4 \%$ for Ajisai. The data were collected at the following satellite distance ranges: from 1600 to $2500 \mathrm{~km}$ (Ajisai, 12.07.2015, h 3.42 CEST), from 1100 to $1500 \mathrm{~km}$ (Stella, 12.07.2015, h 3.08 CEST), and from 1200 to $1500 \mathrm{~km}$ (Beacon-C, 11.07.2015, h 1.33 CEST), giving two-way channel lengths ranging from 2200 up to $5000 \mathrm{~km}$. The interference patterns in Fig. 4 clearly demonstrate that the coherence between the two temporal modes is preserved along these thousand kilometer scale channels with rapidly moving retroreflectors. We attribute the different visibilities to residual vibrations of the unbalanced MZI between the upgoing and downgoing pulses, since the intrinsic visibility of the interferometer was measured to be above $95 \%$. To improve the visibility it would be necessary to redesign the full interferometric setup to further mitigate this effect. We note that, in the doublepass configuration, the interferometer is sensitive to vibrations with frequencies higher than $1 / \mathrm{rtt}$ ( $\mathrm{rtt}$ is the round trip time). Indeed, the lower visibility is obtained with Ajisai, the satellite with the larger distance from the ground (from 1600 to $2000 \mathrm{~km}$ ), with a rtt between 10.7 and $16.7 \mathrm{~ms}$. For the other two satellites the rtt is typically lower than $10 \mathrm{~ms}$.

The mean number of photons $\mu$ in the received pulses may be derived by measuring the detection rate and using the optical losses $\eta \sim 0.27$ in the receiving setup. At the primary mirror of the receiving telescope, the averages $\mu$ during the data acquisition are given by $\mu \approx 7 \times 10^{-4}$ for Beacon-C, $\mu \approx 2 \times 10^{-3}$ for Ajisai, and $\mu \approx 9 \times 10^{-4}$ for Stella. From these values we may conclude that interference was probed at the single photon level. Indeed, the probability of having more that one photon per pulse in the receiver MZI is $\eta \mu^{2} / 2$. We estimated that the mean number of photons $\mu_{\text {sat }}$ leaving from the satellites is $\mu_{\text {sat }}^{\text {(Stella) }}<20$ and $\mu_{\text {sat }}^{\text {(Ajisai) }}<60$ [23] (the instantaneous values fluctuate due to pointing error and turbulence) with a total downlink attenuation between 60 and $70 \mathrm{~dB}$ (including the total detection setup losses $\eta_{r x} \sim 20 \mathrm{~dB}$ due to the optical losses $\eta \sim 0.27$ and the fact that we used a single photomultiplier tube with a $10 \%$ efficiency at the output of the MZI).

Conclusions.-Interference at the single photon level between two temporal modes was observed along a path that includes a rapidly moving retroreflector on a satellite and with a length up to $5000 \mathrm{~km}$. We have experimentally demonstrated that the relative motion of the satellite with respect to the ground induces a varying phase that modulates the interference pattern. This varying phase is not present in the case of fixed terminals. The effect resulted from the measured interference pattern during passages of three satellites, Beacon-C, Stella, and Ajisai, having different relative velocities and distances from the MLRO ground station.

Up to now, photon polarization was the only degree of freedom exploited in long-distance free-space quantum communications, along ground links of $143 \mathrm{~km}$ [24-26] or in efforts towards quantum key distribution in space channels, of a length as large as $2000 \mathrm{~km}$, as recently demonstrated by our group [22]. Indeed, time-bin encoding was never implemented for quantum communication over long-distance free-space channels, fearing that turbulence effects on the wave front might spoil the interference. Here, we have demonstrated that atmospheric turbulence is not detrimental for time-bin encoding in long-distance free-space propagation. Indeed, the two temporal modes separated by a few nanoseconds are identically distorted by the propagation in turbulent air, whose dynamics is in the millisecond scale [26]: the key point here is the careful matching of the interfering wave fronts in the two arms, as shown in Fig. 1. The results here presented attest to the feasibility of the time-bin or phase encoding technique in the context of space quantum communications.

Furthermore, the measurement of interference in space is a milestone to investigate one of the big unresolved puzzle 
in physics, namely, the interplay of quantum theory with gravitation. As recently proposed theoretically by M. Zych et al. $[27,28]$ [the optical version of the original ColellaOverhauser-Werner (COW) experiment realized with neutrons [29] ], interference with single photons in space is a witness of general relativistic effects: the gravitational phase shift between a superposition of two photon wave packets could be highlighted in the context of a large distance quantum optics experiment. In the case of our setup, the gravitational shift for the Ajisai satellite corresponds to about 2 mrad [see Eq. (23) of Ref. [28] ]. We point out that, unlike the case of effects manifested by photon polarization rotation, such small gravitational effects may be better highlighted by using true single photons and by increasing the temporal separation of the two interfering modes (with an increase in the difficulty in stabilizing the interferometers with a large imbalancement). To reveal the effect of gravity in quantum experiments several other proposals have been presented [28]: these include the exchange of elementary particles from moving and accelerated reference frames, which would allow to test Bell's inequalities [30,31] and wave function collapse and possible gravity-induced decoherence [32] in laser interferometry with a long baseline.

The interference patterns measured in the present experiment demonstrate that a coherent superposition between two temporal modes holds in the photon propagation and its interference can be indeed observed over very long channels involving moving terminals at a fast relative velocity. We believe that the results here described attest to the viability of the use of temporal modes of light for fundamental tests of physics and quantum communications around the planet and beyond.

We would like to thank Francesco Schiavone, Giuseppe Nicoletti, and the MRLO technical operators for collaboration and support, Professor Roberto Regazzoni of INAFOsservatorio Astronomico di Padova for the useful discussion on the interferometer optics, as well as Dr. Davide Bacco and Simone Gaiarin for their contributions to the setup. We also thank Franco Ambrico for the image of the MLRO. Our work was supported by the StrategicResearch-Project QUINTET of the Department of Information Engineering, University of Padova, and the Strategic-Research-Project QUANTUMFUTURE of the University of Padova.

Note added.-Recently, a laboratory experiment for exploiting time-bin encoding after free-space propagation was reported [33].

*paolo.villoresi@dei.unipd.it

[1] N. Bohr, Can quantum-mechanical description of physical reality be considered complete?, Phys. Rev. 48, 696 (1935).
[2] G. I. Taylor, Interference fringes with feeble light, Proc. Cambridge Philos. Soc. 15, 114 (1909).

[3] P. Grangier, A. Aspect, and J. Vigue, Quantum Interference Effect for Two Atoms Radiating a Single Photon, Phys. Rev. Lett. 54, 418 (1985).

[4] C. Jönsson, Electron diffraction at multiple slits, Am. J. Phys. 42, 4 (1974).

[5] H. Rauch and S. A. Werner, Neutron Interferometry (Oxford University Press, Oxford, 2015).

[6] S. Eibenberger, S. Gerlich, M. Arndt, M. Mayor, and J. Tüxen, Matter-wave interference of particles selected from a molecular library with masses exceeding 10,000 amu, Phys. Chem. Chem. Phys. 15, 14696 (2013).

[7] B. Korzh, C. C. W. Lim, R. Houlmann, N. Gisin, M. J. Li, D. Nolan, B. Sanguinetti, R. Thew, and H. Zbinden, Provably secure and practical quantum key distribution over $307 \mathrm{~km}$ of optical fibre, Nat. Photonics 9, 163 (2015).

[8] J. Aasi et al., Advanced LIGO, Class. Quantum Grav. 32, 074001 (2015).

[9] B. E. A. Saleh and M. C. Teich, Fundamentals of Photonics (Wiley, New York, 1991).

[10] J. D. Franson, Bell Inequality for Position and Time, Phys. Rev. Lett. 62, 2205 (1989).

[11] W. Tittel, J. Brendel, H. Zbinden, and N. Gisin, Violation of Bell Inequalities by Photons More than $10 \mathrm{Km}$ Apart, Phys. Rev. Lett. 81, 3563 (1998).

[12] G. Lima, G. Vallone, A. Chiuri, A. Cabello, and P. Mataloni, Experimental Bell-inequality violation without the postselection loophole, Phys. Rev. A 81, 040101 (2010).

[13] G. Carvacho, J. Cariñe, G. Saavedra, Á. Cuevas, J. Fuenzalida, F. Toledo, M. Figueroa, A. Cabello, J.-Å. Larsson, P. Mataloni, G. Lima, and G. B. Xavier, Postselection-Loophole-Free Bell Test Over an Installed Optical Fiber Network, Phys. Rev. Lett. 115, 030503 (2015).

[14] V. Scarani, H. Bechmann-Pasquinucci, N. Cerf, M. Dušek, N. Lütkenhaus, and M. Peev, The security of practical quantum key distribution, Rev. Mod. Phys. 81, 1301 (2009).

[15] D. Bacco, M. Canale, N. Laurenti, G. Vallone, and P. Villoresi, Experimental quantum key distribution with finite-key security analysis for noisy channels, Nat. Commun. 4, 2363 (2013).

[16] A. Muller, T. Herzog, B. Huttner, W. Tittel, H. Zbinden, and N. Gisin, Plug and play systems for quantum cryptography, Appl. Phys. Lett. 70, 793 (1997).

[17] J. Brendel, N. Gisin, W. Tittel, and H. Zbinden, Pulsed Energy-Time Entangled Twin-Photon Source for Quantum Communication, Phys. Rev. Lett. 82, 2594 (1999).

[18] N. Gisin, G. Ribordy, W. Tittel, and H. Zbinden, Quantum cryptography, Rev. Mod. Phys. 74, 145 (2002).

[19] I. Ali-Khan, C. J. Broadbent, and J. C. Howell, LargeAlphabet Quantum Key Distribution using Energy-Time Entangled Bipartite States, Phys. Rev. Lett. 98, 060503 (2007).

[20] See Supplemental Material at http://link.aps.org/ supplemental/10.1103/PhysRevLett.116.253601, for details about the experimental design, the technique used for the temporal synchronization and the detailed calculations leading to equations (1) and (2) of the main text. 
[21] P. Villoresi, T. Jennewein, F. Tamburini, M. Aspelmeyer, C. Bonato, R. Ursin, C. Pernechele, V. Luceri, G. Bianco, A. Zeilinger, and C. Barbieri, Experimental verification of the feasibility of a quantum channel between space and Earth, New J. Phys. 10, 033038 (2008).

[22] G. Vallone, D. Bacco, D. Dequal, S. Gaiarin, V. Luceri, G. Bianco, and P. Villoresi, Experimental Satellite Quantum Communications, Phys. Rev. Lett. 115, 040502 (2015).

[23] The technique used for the estimation of $\mu_{\text {sat }}$ is not applicable for the Beacon-C satellite, due to the lack of data (it was launched on 1965). We note that its distance to the ground is similar to that of the Stella satellite and a similar $\mu_{\text {sat }}$ could be expected.

[24] R. Ursin et al., Entanglement-based quantum communication over 144 km, Nat. Phys. 3, 481 (2007).

[25] X.-S. Ma et al., Quantum teleportation over 143 kilometres using active feed-forward, Nature (London) 489, 269 (2012).

[26] I. Capraro, A. Tomaello, A. Dall'Arche, F. Gerlin, R. Ursin, G. Vallone, and P. Villoresi, Impact of Turbulence in Long Range Quantum and Classical Communications, Phys. Rev. Lett. 109, 200502 (2012).

[27] M. Zych, F. Costa, I. Pikovski, and Č. Brukner, Quantum interferometric visibility as a witness of general relativistic proper time, Nat. Commun. 2, 505 (2011).
[28] D. Rideout, T. Jennewein, G. Amelino-Camelia, T. F. Demarie, B. L. Higgins, A. Kempf, A. Kent, R. Laflamme, X. Ma, R. B. Mann, E. Martín-Martínez, N. C. Menicucci, J. Moffat, C. Simon, R. Sorkin, L. Smolin, and D. R. Terno, Fundamental quantum optics experiments conceivable with satellites reaching relativistic distances and velocities, Classical Quantum Gravity 29, 224011 (2012).

[29] R. Colella, A. W. Overhauser, and S. Werner, Observation of Gravitationally Induced Quantum Interference, Phys. Rev. Lett. 34, 1472 (1975).

[30] J.S. Bell, On the Einstein-Podolsky-Rosen paradox, Physics (Long Island City, N.Y.) 1, 195 (1964).

[31] J. F. Clauser, M. A. Horne, A. Shimony, and R. A. Holt, Proposed Experiment to Test Local Hidden-Variable Theories, Phys. Rev. Lett. 23, 880 (1969).

[32] G. Ghirardi, R. Grassi, and A. Rimini, Continuousspontaneous-reduction model involving gravity, Phys. Rev. A 42, 1057 (1990).

[33] J. Jin, S. Agne, J.-P. Bourgoin, Y. Zhang, N. Lütkenhaus, and T. Jennewein, Efficient time-bin qubit analyzer compatible with multimode optical channels, arXiv: 1509.07490. 